\title{
Gamma-ray signatures of Dark Matter
}

\author{
Marco CIRELLI ${ }^{1}$ a \\ ${ }^{1}$ Laboratoire de Physique Théorique et Hautes Energies (LPTHE), \\ UMR 7589 CNRS \& UPMC, \\ 4 Place Jussieu, F-75252, Paris, France
}

\begin{abstract}
I review the ways in which Dark Matter can produce gamma-rays (and lower energy photons) and I apply the considerations to three specific examples: the tests of multi-TeV pure-WIMP candidates based on high energy gamma-rays from the Galactic Center or dwarfs, the importance of bremsstrahlung emission for light DM, the updated constraints on DM from all-sky radio surveys of the Milky Way.
\end{abstract}

\section{Introduction}

Cosmology and astrophysics provide several convincing evidences of the existence of Dark Matter (DM). The observation that some mass is missing to explain the internal dynamics of galaxy clusters and the rotations of galaxies dates back respectively to the ' 30 s and the ' $70 \mathrm{~s}$. The observations from weak lensing, for instance in the spectacular case of the so-called 'bullet cluster', provide evidence that there is mass where nothing is optically seen. More generally, global fits to a number of cosmological datasets (Cosmic Microwave Background, Large Scale Structure and also Type Ia Supernovae) allow to determine very precisely the amount of DM in the global energy-matter content of the Universe at $\Omega_{\mathrm{DM}} h^{2}=0.1188 \pm 0.0010[1]^{1}$.

All these signals pertain to the gravitational effects of Dark Matter at the cosmological and extragalactical scale. Searches for explicit manifestation of the DM particles that are supposed to constitute the halo of our own galaxy (and the large scale structures beyond it) have instead so far been giving negative results, but this might be on the point of changing.

Indirect searches for DM are of particular interest. These searches aim at detecting the signatures of the annihilations or decays of DM particles in the fluxes of Cosmic Rays (CRs), intended in a broad sense: charged particles (electrons and positrons, antiprotons, antideuterium), photons (gamma rays, X-rays, synchrotron radiation), neutrinos. In this contribution I am going to focus in particular on a few examples involving photon signals, from the high energy (multi-TeV gamma rays) to the low energy ones (radio-waves). Before discussing the examples, though, let's review generically how Dark Matter produces photons.

\footnotetext{
a e-mail: marco.cirelli@gmail.com

${ }^{1}$ Here $\Omega_{\mathrm{DM}}=\rho_{\mathrm{DM}} / \rho_{c}$ is defined as usual as the energy density in Dark Matter with respect to the critical energy density of the Universe $\rho_{c}=3 H_{0}^{2} / 8 \pi G_{N}$, where $H_{0}$ is the present Hubble parameter. $h$ is its reduced value $h=H_{0} / 100 \mathrm{~km} \mathrm{~s}^{-1} \mathrm{Mpc}^{-1}$.
} 


\section{Indirect DM detection via gamma-rays}

In general, DM annihilation (or decay) can produce photon fluxes in many ways, among which:

I) 'Prompt' gamma-rays: produced directly by DM annihilations themselves. In turn, however, these gamma-rays can originate from different stages of the annihilation process:

Ia) From the bremsstrahlung of charged particles and the fragmentation of hadrons, e.g. $\pi^{0}$, in the final states of the annihilations. These processes generically give origin to a continuum of $\gamma$-rays which peaks at energies somewhat smaller than the DM mass $m_{\mathrm{DM}}$, i.e. typically in the range of tens of $\mathrm{GeV}$ to multi-TeV. The spectra can be computed in a model independent way (see e.g. [2]), since all one needs to know is the pair of primary SM particles.

Ib) From the bremsstrahlung from one of the internal particles in the annihilation diagram. This typically gives rise to a sharp feature that peaks at an energy corresponding to the DM mass. The process is in general subdominant with respect to the continuum, but it can be particularly important in cases in which the continuum itself is suppressed by some mechanism, e.g. helicity constraints, which are lifted by the internal radiation. The spectrum from this contribution cannot be computed without knowing the details of the annihilation model.

Ic) From an annihilation directly into a pair of gamma-rays, which gives rise to a line spectrum at the energy corresponding to the mass of the DM. Since DM is neutral, this annihilation has to proceed via some intermediation (typically a loop of charged particles) and it is therefore suppressed by (typically) 2 to 4 orders of magnitude.

In any case, since these $\gamma$-rays originate directly from the annihilations themselves, their spatial distribution follows closely the distribution of DM.

II) Secondary radiation, emitted by the $e^{+} e^{-}$, which have been produced by the annihilation process, when they interact with the environment:

IIa) ICS gamma-rays: produced by the Inverse Compton Scattering (ICS) of the energetic electrons and positrons, created in the DM annihilation, onto the low energy photons of the CMB, the galactic star-light and the infrared-light, which are thus upscattered in energy. Typically, they cover a wider range of energies than prompt gamma rays, from energies of a fraction of the DM mass to almost up to the DM mass itself. Their spatial distribution traces the distribution of $e^{ \pm}$, which originate from DM but then diffuse out in the whole galactic halo (as seen above).

IIb) Bremsstrahlung gamma-rays: produced by the same energetic electrons and positrons onto the gas in the interstellar medium. Typically, they are of lower energy than ICS rays; their spatial distribution traces the $e^{ \pm}$but also the density of target gas, so it is typically concentrated in the galactic disk.

IIc) Synchrotron emission: consisting in the radiation emitted in the magnetic field of the Galaxy by the $e^{ \pm}$produced by DM annihilations. For an intensity of the magnetic field of $O(\mu \mathrm{Gauss})$, like in the case of the Milky Way halo, and for $e^{ \pm}$of GeV-TeV energies, the synchrotron emission falls in the $\mathrm{MHz}-\mathrm{GHz}$ range, i.e. in the radio band. For large magnetic fields and large DM masses it can reach up to $\mathrm{EHz}$, i.e. the $\mathrm{X}$-ray band. Their region of origin is necessarily concentrated where the magnetic field is highest; in particular the GC is the usual target of choice. However, it has been recently suggested that the galactic halo at large, or even the extragalactic ones, can be interesting sources, as we will discuss below.

Individuating the best targets to search for these annihilation signals is one of the main games in the field. Not very surprisingly, the preferred targets have to be (i) regions with high DM densities and/or 
(ii) regions where the astrophysical 'background' is reduced and therefore the signal/noise ratio is favorable. The distinction between (i) and (ii) is of course not clear-cut, and of course there are specific cases in which other environmental reasons make a region more suitable than another (such as in the case of synchrotron radiation which needs a region with a strong magnetic field). Moreover, new promising targets keep being individuated. However, for the sake of schematizing, one can list the following targets at which the experiments look:

- The Milky Way Galactic Center (GC) - (i).

- Small regions around or just outside the GC, such as the Galactic Center Halo $(\mathrm{GCH}$, an annulus of about $1^{\circ}$ around the GC, excluding the Galactic Plane) etc - (i) + (ii).

- Wide regions of the Galactic Halo $(\mathrm{GH})$ itself (such as observational windows centered at the GC and several tens of degrees wide in latitude and longitude, from which a diffuse flux of gamma-rays is expected, including the one due to the ICS emission from the diffused population of $e^{ \pm}$from DM annihilations - (ii).

- Globular clusters (GloC), which are dense agglomerates of stars, embedded in the Milky Way galactic halo. They are a peculiar kind of target since they are not supposed to be DM dominated, quite the opposite, as they are rich of stars. The interest in them arises from two facts: that they may have formed inside a primordial DM subhalo and some of the DM may have remained trapped; that the density of baryonic matter may create by attraction a DM spike and thus enhance the annihilation flux- (i).

- Subhalos of the galactic DM halo, whose position, however, is of course unknown a priori.

- Satellite galaxies of the Milky Way, often of the dwarf spheroidal (dSph) class, such as Sagittarius, Segue1, Draco and several others, which are star-deprived and believed to be DM dominated - (i) + (ii). The 'problem' with dSphs is precisely that the determination of their DM content and distribution relies on stars as kinematical tracers and therefore suffers from rather large uncertainties.

- Large scale structures in the relatively nearby Universe, such as galaxy clusters (e.g. the Virgo, Coma, Fornax, Perseus clusters, and several others with catalog names that are less pleasant to write) - (i) + (ii)

- The Universe at large, meaning looking at the isotropic flux of (redshifted) $\gamma$-rays that come to us from DM annihilation in all halos and all along the recent history of the Universe. Often this flux is called 'extragalactic' or 'cosmological' - (ii)

Let us now move to discuss a few examples of how photon searches can play a relevant role in the Dark Matter parameter space, in very different contexts.

\section{Testing pure WIMP multi-TeV DM models with high energy gamma rays}

In this section we summarize the findings of [3]: we start by reconsidering the model of Minimal Dark Matter (a fermionic, hypercharge-less quintuplet of the EW interactions) and compute its gamma ray signatures. We compare them with a number of gamma ray probes. Those that turn out to be most significant are the galactic halo diffuse measurements, the galactic center line searches and recent dwarf galaxies observations. We find that the original minimal model, whose mass is fixed at $9.4 \mathrm{TeV}$ by the relic abundance requirement, is constrained by the line searches from the Galactic Center: it is ruled out if the Milky Way possesses a cuspy profile such as NFW but it is still allowed if it has a cored one. This is illustrated by fig. 1, right panel. Observations of dwarf spheroidal galaxies are also relevant, and ongoing astrophysical progresses on these systems have the potential to eventually rule 

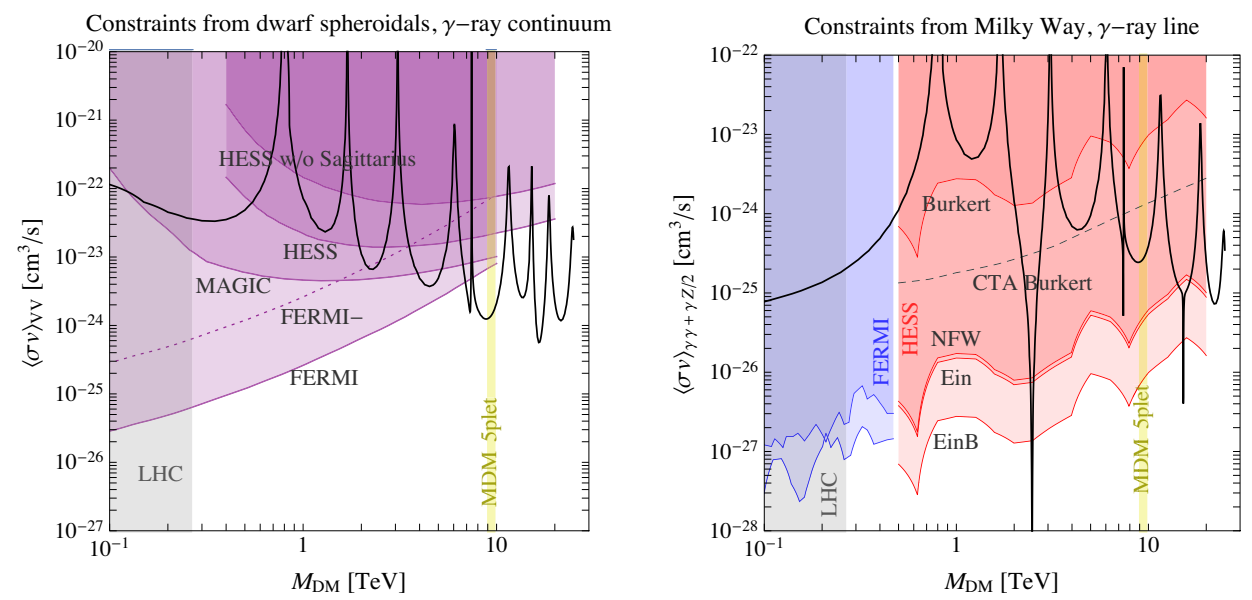

Figure 1. Constraints on the DM annihilation cross section into gauge bosons from dwarf spheroidal galaxies (left) and the Milky Way (right). Figure from [3].

out the model. As the figures show, we also explore a wider mass range, which applies to the case in which the relic abundance requirement is relaxed. Our constraints can therefore be applied to generic $\mathrm{DM}$ candidates with a mass in the multi-TeV range which annihilate into gauge bosons.

\section{Bremsstrahlung gamma-rays from low mass DM}
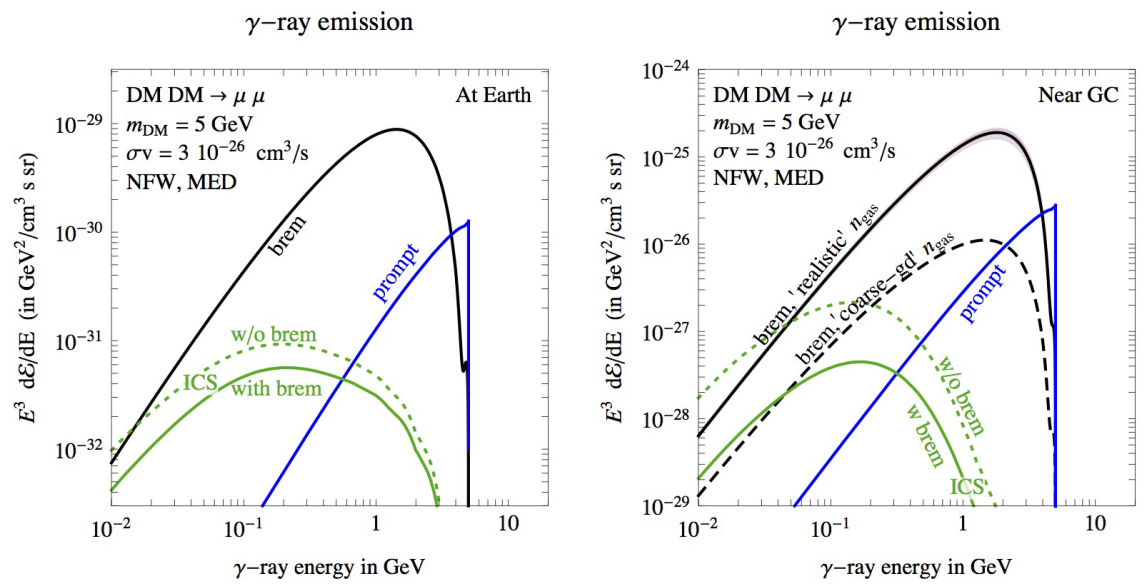

Figure 2. Full gamma-ray emission from light DM annihilations, including the bremsstrahlung contribution (and the modification to the Inverse Compton contribution due to taking into account bremsstrahlung), for the conditions typical of the location of the Earth (left) or of the Galactic Center (right). Figure from [4].

This section is based on the results in [4]. We stress the often-neglected role of bremsstrahlung processes on the interstellar gas in computing indirect signatures of DM annihilation in the Galaxy, 
particularly for light DM candidates in the phenomenologically interesting $O(10) \mathrm{GeV}$ mass range. Especially from directions close to the Galactic Plane, the gamma-ray spectrum is altered via two effects: directly, by the photons emitted in the bremsstrahlung process by energetic electrons which are among the DM annihilation byproducts; indirectly, by the modification of the same electron spectrum, due to the additional energy loss process in the diffusion-loss equation (e.g. the resulting inverse Compton emission is altered). Fig. 2 illustrates this point by plotting the total gamma-ray emission from light DM annihilations in conditions that are typical of the region close to the Earth or of the Galactic Center. So, for computing precise spectra in the (sub-)GeV range, it is important to obtain a reliable description of the Galaxy gas distribution as well as to compute self-consistently the gammaray emission and the solution to the diffusion-loss equation.

\section{Constraining DM with galactic radio surveys}
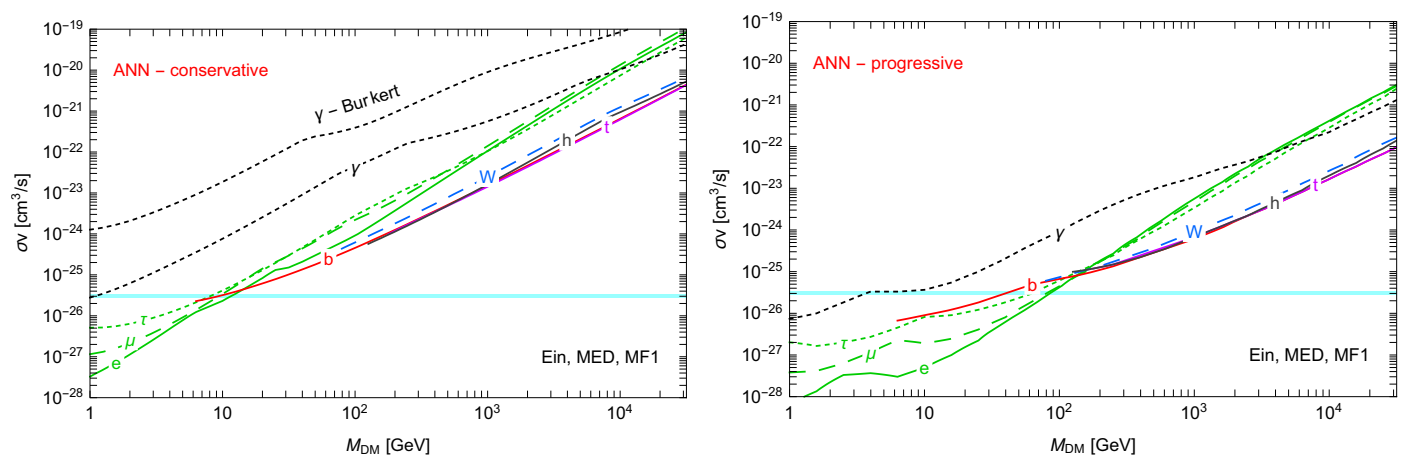

Figure 3. Constraints on DM annihilation from radio surveys, adopting a conservative (left) or progressive (right) approach (see text). Figure from [5].

This section is based on the study in [5], where we perform a detailed analysis of the synchrotron signals produced by dark matter annihilations (and decays). We consider different set-ups for the propagation of electrons and positrons, the galactic magnetic field and dark matter properties. We then confront these signals with radio and microwave maps, including PlancK measurements, from a frequency of $22 \mathrm{MHz}$ up to $70 \mathrm{GHz}$. We derive two sets of constraints: conservative and progressive. The former is obtained with no assumptions on the astrophysical background and just requires that the DM signal does not exceed the measured intensity. The latter is instead based on a modeling of the astrophysical emission: reducing the room avaiilable for DM, it leads to more stringent constraints. The results are depicted in fig. 3, for different annihilation channels. In general, radio and microwave constraints are complementary to those obtained with other indirect detection methods, especially for dark matter annihilating into leptonic channels.

\section{Conclusions}

In this contribution I have tried to review the different ways in which galactic gamma rays and radio surveys can help in the search for Dark Matter signatures. I first considered the searches of high energy gamma-rays, by FeRMI, Hess and the other Cherenkov telescopes, as a test for models featuring 
pure-WIMP multi-TeV DM candidates, such as for instance the Minimal Dark Matter model. Then I stressed the importance of including bremsstrahlung as a process for the energy losses suffered by the electrons and positrons produced by DM in the galaxy, especially close to the galactic plane where the density of gas is sizable, and therefore its importance for the computation of DM secondary radiation. Finally, I presented the results of a new evaluation of the constraints on DM from all-sky radio surveys of the Milky Way.

As a general rule, DM indirect searches, and a fortiori gamma-ray searches, are complementary to one another, for the different annihilation channels, different chosen targets and different energy regimes. Fig. 4 collects some of the most relevant constraints, for the case of the $b \bar{b}$ channel.

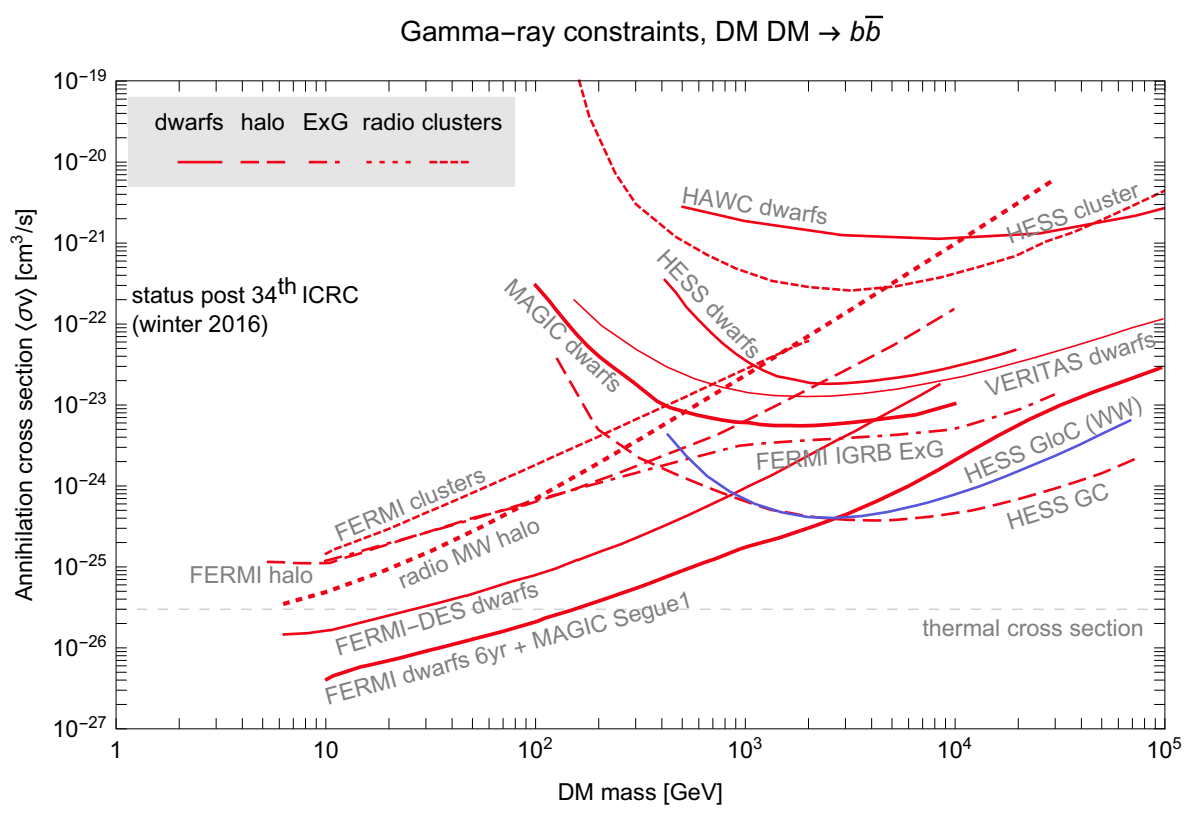

Figure 4. Bounds on DM annihilation imposed by different gamma-ray (and radio) observations. Figure from [6].

\section{References}

[1] P. A. R. Ade et al. [Planck Coll.], arXiv:1502.01589 [astro-ph.CO].

[2] M. Cirelli et al., JCAP 1103 (2011) 051 [JCAP 1210 (2012) E01] [arXiv:1012.4515 [hep-ph]].

[3] M. Cirelli, T. Hambye, P. Panci, F. Sala and M. Taoso, JCAP 1510 (2015) no.10, 026 doi:10.1088/1475-7516/2015/10/026 [arXiv:1507.05519 [hep-ph]].

[4] M. Cirelli, P. D. Serpico and G. Zaharijas, JCAP 1311 (2013) 035 doi:10.1088/14757516/2013/11/035 [arXiv:1307.7152 [astro-ph.HE]].

[5] M. Cirelli and M. Taoso, JCAP 1607 (2016) no.07, 041 doi:10.1088/1475-7516/2016/07/041 [arXiv:1604.06267 [hep-ph]].

[6] M. Cirelli, arXiv:1511.02031 [astro-ph.HE]. 